\title{
An extended mode-matching model for improved relative permittivity measurements using a split-cylinder resonator
}

\author{
S. Zinal and U. Arz \\ Physikalisch-Technische Bundesanstalt (PTB), Bundesallee 100, 38116 Braunschweig, Germany \\ Correspondence to: S. Zinal (sherko.zinal@ptb.de)
}

Received: 20 December 2013 - Accepted: 7 September 2014 - Published: 11 November 2014

\begin{abstract}
In this paper we present an extended modematching model for a split-cylinder resonator involving $T E$ and $T M$ resonant modes. The new model enhances the known $T E_{0 \text { np }}$ mode-matching model by improving mode identification and distortion analysis and providing better frequency range coverage for relative permittivity estimation. In order to verify the new model, measured spectra of several substrate materials are analyzed and the estimation results are compared with those of the $T E_{0 \mathrm{np}}$ mode-matching model.
\end{abstract}

\section{Introduction}

The split-cylinder resonator technique employs a cylindrical cavity which is transversally separated into two halves with a laminar sample material inserted between them as shown in Fig. 1. The feeding structure is realized by a coupling loop in each of the resonator sections exciting preferably $T E$ resonant modes, the resonance frequency and quality factor of which is used to determine the relative permittivity and the loss tangent of the tested material.

This technique was first proposed in Kent (1988) as a nondestructive method for measuring the complex permittivity of low loss substrate materials utilizing only the $T E_{011}$ mode.

A considerable improvement of the method was achieved in Janezic and Baker-Jarvis (1999) and Janezic et al. (2004) by developing a theoretical model which includes general odd-numbered $T E_{0 n p}$ modes and thus, on the one hand, allows broadband permittivity and loss tangent measurements and, on the other hand, considers the fringing fields in the gap region minimizing systematic errors.
The main practical difficulty encountered applying this method is the precise identification of the desired resonant mode in the frequency spectrum if the relative permittivity of the tested material is not sufficiently known. Especially at high frequencies where the higher order modes occur very close to each other, the mode identification can become a trial and error procedure. To overcome this problem a pre-estimation of the relative permittivity was suggested in Janezic et al. (2009) by utilizing the $T E_{111}$ mode, which has the lowest resonance frequency among the $T E$ modes and can be easily identified even for test materials with unknown relative permittivities.

A further problem encountered in measurements is the prediction of resonant mode distortion, which is caused by coupling or overlapping of the desired $T E_{0 \mathrm{np}}$ mode with other modes. This problem cannot be handled with a model confined to the $T E_{0 \text { np }}$ mode family. In the best of such cases a considerable deviation of the determined relative permittivity or loss tangent from the expected values can indicate the distortion. However, a tunable split-cylinder resonator, presented in Fang et al. (2004), can be used to shift the desired mode out of the overlapping region by adjusting the distance of the resonator endplates.

To overcome the problems mentioned above, a new modematching model for the split-cylinder resonator is presented in this paper. Even though some simplifications are applied, the developed model extends the model presented in Janezic et al. (2004) by including general $T E_{\mathrm{mnp}}$ modes as well as general $T M_{\mathrm{mnp}}$ modes.

\section{Theoretical model}

In this section the extended mode-matching model will be formulated for the split-cylinder resonator shown in Fig. 1. 


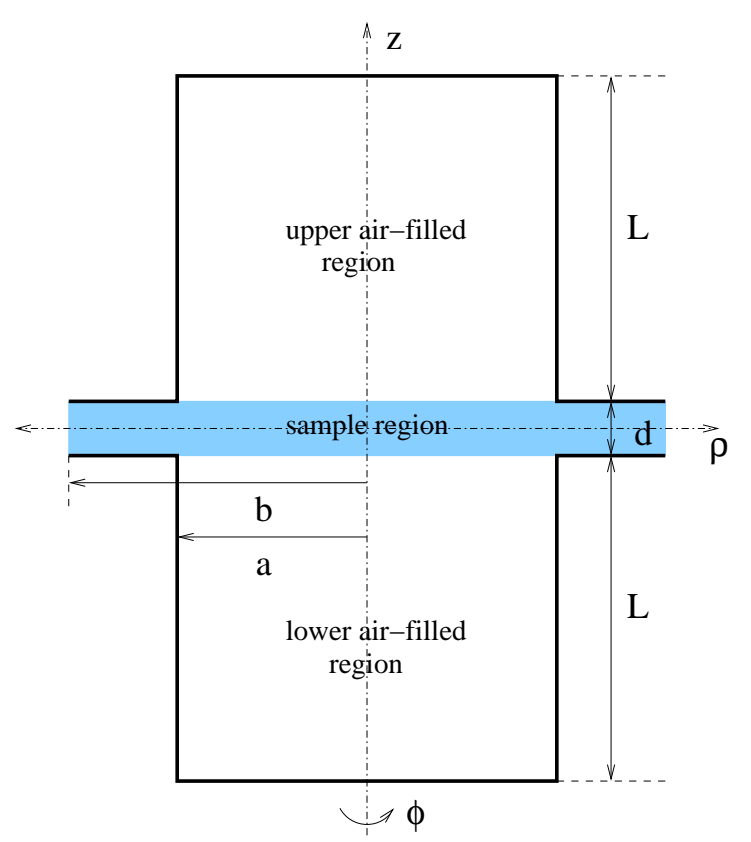

Figure 1. Geometrical dimensions of the split-cylinder resonator.

\subsection{Preliminary remarks}

Although the feeding structure, formed as small loops with axis in $z$ direction, is intended to excite only $T E$ resonant modes, feeding impurities and the discontinuity at the gap edges give rise to longitudinal ( $z$-directed) electric field components and consequently cause $T M$ modes to be excited. Due to their purely edge-tangential electric field, only the $T E_{0 \text { np }}$ modes do not cause any longitudinal electric field component at the gap discontinuity.

The $T E_{0 \mathrm{np}}$ modes, which have only a one-dimensional $(\rho-)$ dependency over the transverse cross-section, are suitable for superposition of higher $n$-order modes for matching boundary conditions at the substrate surface. Thus the mode-matching model presented in Janezic et al. (2004) (see Janezic, 2003 for more details) allows to consider the fringing fields in the gap region. The other $T E_{\mathrm{mnp}}$ modes, however, have a two-dimensional ( $\rho$ - and $\phi$-) dependency over the transverse cross-section and are therefore not applicable to superposition of higher $m n$-order modes for matching boundary conditions at the substrate surface.

Rather, the present approach confines the boundary condition at the substrate surface to be matched by a single mode on each side. Subsequently, since the step discontinuity at the gap edge cannot be fitted by a single mode, it is necessary to set $b=a$ neglecting the fringing fields in the gap region. With respect to these simplifications a similar approach utilizing the $T E_{111}$ mode for nondestructive permittivity measurements was presented in Ali et al. (2006).

The procedure distinguishes between modes with odd or$\operatorname{der}(p=1,3,5, \ldots)$ and even order $(p=2,4,6, \ldots)$ in $z$ di- rection. Due to the symmetry in $z$ direction it is sufficient to treat only one half of the resonator, e.g. from $z=0$ to $z=L+d / 2$.

\subsection{Formulating the electromagnetic fields}

The transversal electromagnetic fields in the upper air-filled region (subscript $u$ ) can be written as:

$$
\begin{aligned}
E_{\rho_{u}}= & A_{m n}^{\mathrm{te}} \frac{m}{h_{m n_{u}}^{\mathrm{te}} \rho} J_{m}\left(h_{m n_{u}}^{\mathrm{te}} \rho\right) \sin (m \phi) \sin \left[p_{m n_{u}}^{\mathrm{te}}\left(L+\frac{d}{2}-z\right)\right]+ \\
E_{\phi_{u}}= & A_{m n}^{\mathrm{tm}} p_{m n_{u}}^{\mathrm{tm}} J_{m}^{\prime}\left(h_{m n_{u}}^{\mathrm{tm}} \rho\right) \sin (m \phi) \sin \left[p_{m n_{u}}^{\mathrm{tm}}\left(L+\frac{d}{2}-z\right)\right] \\
H_{\rho_{u}}= & \left.\frac{-1}{j \omega \mu_{0}} A_{m n}^{\mathrm{te}} \rho\right) \cos (m \phi) \sin \left[p_{m n_{u}}^{\mathrm{te}}\left(L+\frac{d}{2}-z\right)\right]+ \\
& \frac{k_{u}^{2}}{j \omega n_{u}} A_{m n}^{\mathrm{tm}} \frac{m p_{m n_{u}}^{\mathrm{tm}}}{h_{m}^{\mathrm{tm}}} J_{m}^{\prime}\left(h_{m n_{u}}^{\mathrm{tm}}\left(h_{m n_{u}}^{\mathrm{tm}} \rho\right) \cos (m \phi) \sin \left[p_{m n_{u}}^{\mathrm{tm}}\left(L+\frac{d}{2}-z\right)\right]\right. \\
h_{m n_{u}}^{\mathrm{tm}} \rho & \left.J_{m n_{u}}^{\mathrm{tm}} \rho\right) \cos (m \phi) \cos \left[p_{m n_{u}}^{\mathrm{te}}\left(L+\frac{d}{2}-z\right)\right]- \\
H_{\phi_{u}}= & \frac{1}{j \omega \mu_{0}} A_{m n}^{\mathrm{te}} \frac{m p_{m n_{u}}^{\mathrm{te}}}{h_{m n_{u}}^{\mathrm{te}} \rho} J_{m}\left(h_{m n_{u}}^{\mathrm{te}} \rho\right) \sin (m \phi) \cos \left[p_{m n_{u}}^{\mathrm{tm}}\left(L+\frac{d}{2}-z\right)\right](3) \\
& \frac{k_{u}^{2}}{j \omega \mu_{0}} A_{m n}^{\mathrm{tm}} J_{m}^{\prime}\left(h_{m n_{u}}^{\mathrm{tm}} \rho\right) \sin (m \phi) \cos \left[p_{m n_{u}}^{\mathrm{tm}}\left(L+\frac{d}{2}-z\right)\right]+
\end{aligned}
$$

The separation constants are defined as follows:

$\left(p_{m n_{u}}^{\mathrm{te}, \mathrm{tm}}\right)^{2}=k_{u}^{2}-\left(h_{m n_{u}}^{\mathrm{te}, \mathrm{tm}}\right)^{2}, h_{m n_{u}}^{\mathrm{te}}=\frac{j_{m, n}^{\prime}}{a}, h_{m n_{u}}^{\mathrm{tm}}=\frac{j_{m, n}}{a}$

where $k_{u}=\omega \sqrt{\mu_{0} \varepsilon_{0} \varepsilon_{r_{u}}}$ is the wave number, $j_{m, n}$ and $j_{m, n}^{\prime}$ are the zeroes of the Bessel function and its derivative, respectively.

For the substrate region (subscript $s$ ), the transversal electromagnetic fields are given as:

$$
\begin{aligned}
& E_{\rho_{s}}=B_{m n}^{\mathrm{te}} \frac{m}{h_{m n_{s}}^{\mathrm{te}} \rho} J_{m}\left(h_{m n_{s}}^{\mathrm{te}} \rho\right) \sin (m \phi)\left\{\begin{array}{c}
\cos \left(p_{m n_{s}}^{\mathrm{te}} z\right) \\
\sin \left(p_{m n_{s}}^{\mathrm{te}} z\right)
\end{array}\right\}+ \\
& B_{m n}^{\mathrm{tm}} p_{m n_{s}}^{\mathrm{tm}} J_{m}^{\prime}\left(h_{m n_{s}}^{\mathrm{tm}} \rho\right) \sin (m \phi)\left\{\begin{array}{c}
\cos \left(p_{m n_{s}}^{\mathrm{tm}} z\right) \\
-\sin \left(p_{m n_{s}}^{\mathrm{tm}} z\right)
\end{array}\right\} \\
& E_{\phi_{s}}=B_{m n}^{\mathrm{te}} J_{m}^{\prime}\left(h_{m n_{s}}^{\mathrm{te}} \rho\right) \cos (m \phi)\left\{\begin{array}{c}
\cos \left(p_{m n_{s}}^{\mathrm{te}} z\right) \\
\sin \left(p_{m n_{s}}^{\mathrm{te}} z\right)
\end{array}\right\}+ \\
& B_{m n}^{\mathrm{tm}} \frac{m p_{m n_{s}}^{\mathrm{tm}}}{h_{m n_{s}}^{\mathrm{tm}} \rho} J_{m}\left(h_{m n_{s}}^{\mathrm{tm}} \rho\right) \cos (m \phi)\left\{\begin{array}{c}
\cos \left(p_{m n_{s}}^{\mathrm{tm}} z\right) \\
-\sin \left(p_{m n_{s}}^{\mathrm{tm}} z\right)
\end{array}\right\} \\
& H_{\rho_{s}}=\frac{-1}{j \omega \mu_{0}} B_{m n}^{\mathrm{te}} p_{m n_{s}}^{\mathrm{te}} J_{m}^{\prime}\left(h_{m n_{s}}^{\mathrm{te}} \rho\right) \cos (m \phi)\left\{\begin{array}{c}
\sin \left(p_{m n_{s}}^{\mathrm{te}} z\right) \\
-\cos \left(p_{m n_{s}}^{\mathrm{te}} z\right)
\end{array}\right\}- \\
& \frac{k_{s}^{2}}{j \omega \mu_{0}} B_{m n}^{\mathrm{tm}} \frac{m}{h_{m n_{s}}^{\mathrm{tm}} \rho} J_{m}\left(h_{m n_{s}}^{\mathrm{tm}} \rho\right) \cos (m \phi)\left\{\begin{array}{c}
\sin \left(p_{m n_{s}}^{\mathrm{tm}} z\right) \\
\cos \left(p_{m n_{s}}^{\mathrm{tm}} z\right)
\end{array}\right\} \\
& H_{\phi_{s}}=\frac{1}{j \omega \mu_{0}} B_{m n}^{\mathrm{te}} \frac{m p_{m n_{s}}^{\mathrm{te}}}{h_{m n_{s}}^{\mathrm{te}} \rho} J_{m}\left(h_{m n_{s}}^{\mathrm{te}} \rho\right) \sin (m \phi)\left\{\begin{array}{c}
\sin \left(p_{m n_{s}}^{\mathrm{te}} z\right) \\
-\cos \left(p_{m n_{s}}^{\mathrm{te}} z\right)
\end{array}\right\}+ \\
& \frac{k_{s}^{2}}{j \omega \mu_{0}} B_{m n}^{\mathrm{tm}} J_{m}^{\prime}\left(h_{m n_{s}}^{\mathrm{tm}} \rho\right) \sin (m \phi)\left\{\begin{array}{c}
\sin \left(p_{m n_{s}}^{\mathrm{tm}} z\right) \\
\cos \left(p_{m n_{s}}^{\mathrm{tm}} z\right)
\end{array}\right\}
\end{aligned}
$$


where the upper and lower terms in the braces describe modes with odd and even $p$ order in $z$ direction, respectively. The separation constants are defined as follows:

$\left(p_{m n_{s}}^{\mathrm{te}, \mathrm{tm}}\right)^{2}=k_{s}^{2}-\left(h_{m n_{s}}^{\mathrm{te}, \mathrm{tm}}\right)^{2}, h_{m n_{s}}^{\mathrm{te}}=\frac{j_{m, n}^{\prime}}{a}, h_{m n_{s}}^{\mathrm{tm}}=\frac{j_{m, n}}{a}$

where $k_{s}=\omega \sqrt{\mu_{0} \varepsilon_{0} \varepsilon_{r_{s}}}$ is the wave number in the substrate region.

\subsection{Formulating the equation system}

The equation system whose solution determines the resonant modes must be solved for odd and even modes separately. In the following we develop the formalism for the case of odd modes. The case of even modes can be treated in a similar manner.

The resonance condition is achieved by enforcing the boundary conditions for the transversal electromagnetic fields at the upper substrate surface $(z=d / 2,0 \leq \rho \leq a)$ :

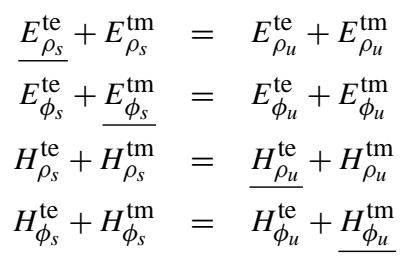

Applying orthogonality relations corresponding to the underlined terms to each of the Eqs. (11)-(14) successively, we get a system of four equations with four unknowns, demonstrated for the first Eq. (11) as follows:

$$
\begin{gathered}
B_{m n}^{\mathrm{te}} \underbrace{\frac{m}{h_{m n_{s}}^{\mathrm{te}}} \cos \left(p_{m n_{s}}^{\mathrm{te}} \frac{d}{2}\right) I_{1}}_{C_{1}}+B_{m n}^{\mathrm{tm}} \underbrace{p_{m n_{s}}^{\mathrm{tm}} \cos \left(p_{m n_{s}}^{\mathrm{tm}} \frac{d}{2}\right) I_{2}}_{C_{2}}= \\
A_{m n}^{\mathrm{te}} \underbrace{\frac{m}{h_{m n_{u}}^{\mathrm{te}}} \sin \left(p_{m n_{u}}^{\mathrm{te}} L\right) I_{3}}_{C_{3}}+A_{m n}^{\mathrm{tm}} \underbrace{p_{m n_{u}}^{\mathrm{tm}} \sin \left(p_{m n_{u}}^{\mathrm{tm}} L\right) I_{4}}_{C_{4}}
\end{gathered}
$$

where $I_{1}-I_{4}$ are integrals over Bessel functions which can be evaluated numerically for a given radius $a$.

Thus evaluating the three remaining equations leads for each combination $(m, n)$ to the following linear system of equations:

$$
\underbrace{\left(\begin{array}{cccc}
C_{1} & C_{2} & -C_{3} & -C_{4} \\
C_{5} & C_{6} & -C_{7} & -C_{8} \\
C_{9} & C_{10} & -C_{11} & -C_{12} \\
C_{13} & C_{14} & -C_{15} & -C_{16}
\end{array}\right)}_{\mathbf{Z}}\left(\begin{array}{c}
B^{\mathrm{te}} \\
B^{\mathrm{tm}} \\
A^{\mathrm{te}} \\
A^{\mathrm{tm}}
\end{array}\right)=0
$$

For the case of even $p$ order we get an analogous system of equations by inserting the corresponding terms from Eqs. (6)-(9).

The linear system of Eqs. (16), describing a resonance condition, has a nontrivial solution only if:

$$
\operatorname{det}[\mathbf{Z}]=0
$$
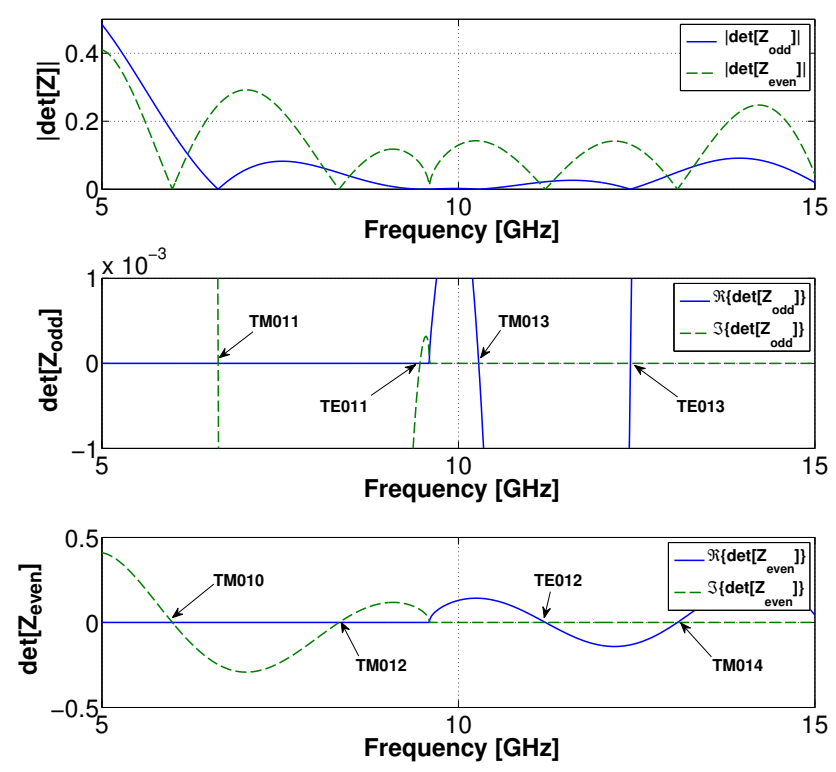

Figure 2. $\operatorname{det}[\mathbf{Z}]$ as a function of frequency for the order combination $(m=0, n=1)$. BF33, $\varepsilon_{r} \approx 4.5, d=0.704 \mathrm{~mm}$.

Equation (17) can be used to calculate either the resonant frequency of the split-cylinder resonator given the substrate permittivity or the substrate permittivity given a measured resonant frequency. To solve Eq. (17) we can take advantage of the peculiarity that $\operatorname{det}[\mathbf{Z}]$ alternates between purely real and purely imaginary values whereas the nonzero part crosses the zero line at the resonance, as demonstrated in Fig. 2 for the order combination $(m=0, n=1)$. This allows for a fast and coarse detection of zeroes over the whole frequency range in a first step. Subsequently, the detected zeroes can be located more accurately by applying root determining methods like the method of false position (regula falsi).

Once the resonant frequencies (or permittivities) have been determined, the coefficients $\left(A^{\mathrm{te}}, A^{\mathrm{tm}}, B^{\mathrm{te}}, B^{\mathrm{tm}}\right)$ for each resonant mode can be calculated by solving Eq. (16) as a singular value decomposition problem. The null space method is not applicable due to rounding errors.

It should be mentioned that accurate root locating is essential for a reliable determination of the mode type ( $T E$ or $T M$ ). Especially modes with order $p=1$ in $z$ direction are very sensitive to inaccuracies in the location of zeroes. Their fields are hyperbolic functions of $z$ in the air-filled region due to an imaginary separation constant $p_{m n_{u}}$, and thus have an evanescent behavior.

\section{Model verification via measurements}

In this section the presented model will be applied to measurement data of several substrate materials to verify the model on the one hand and to demonstrate the advantages provided in measurement data analysis on the other hand. 

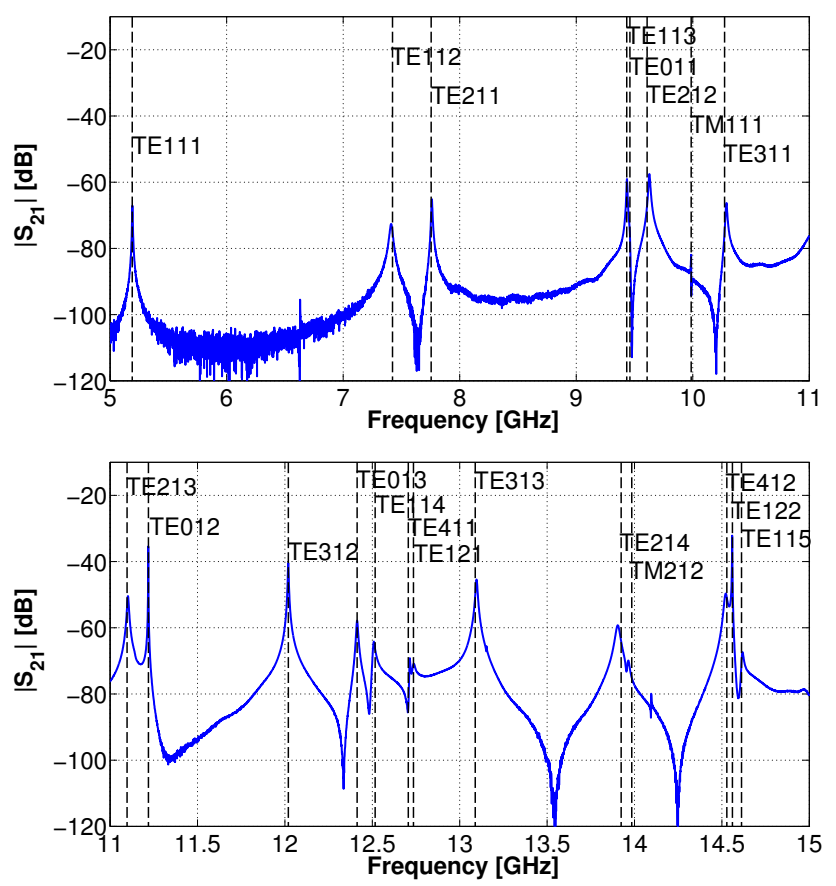

Figure 3. Measured spectrum and predicted resonant modes for $\mathrm{BF} 33, \varepsilon_{r} \approx 4.5, d=0.704 \mathrm{~mm}$.

The split-cylinder resonator used had the dimensions $a=$ $19.053 \mathrm{~mm}$ and $L=25.4 \mathrm{~mm}$.

\subsection{Mode identification in a measured spectrum}

Figure 3 shows the measured spectrum for a BF33 substrate material in the frequency range $5-15 \mathrm{GHz}$ with the resonant modes predicted by the model in this range. The type of the resonant mode ( $T E, T M$ or hybrid) is determined via the coefficient ratios $\left|A^{\mathrm{te}}\right| /\left|A^{\mathrm{tm}}\right|$ and $\left|B^{\mathrm{te}}\right| /\left|B^{\mathrm{tm}}\right|$.

\subsection{Distortion analysis of resonant modes}

Figure 4 shows the measured relative permittivity and loss tangent for a BF33 substrate material applying the $T E_{0 \mathrm{np}}$ mode-matching method presented in Janezic et al. (2004). The obvious deviation at the first measurement point $\left(f_{r}=\right.$ $9.44 \mathrm{GHz}$ ) can be explained with the help of the extended model by predicting a distortion of the $T E_{011}$ mode due to coupling with the $T E_{113}$ mode. In fact, as Fig. 5 demonstrates, the resonant mode evaluated by the measurement algorithm seems to be the $T E_{113}$ mode rather than the desired $T E_{011}$ mode, which is distorted by coupling.

A similar example is shown in Fig. 6 for an AF45 substrate material. In this case the two resonant modes seem to overlap and thus the relative permittivity estimated by the $T E_{0 \mathrm{np}^{-}}$ method does not deviate noticeably. However the distortion affects the measured loss tangent as it is obvious from the results shown in Fig. 7.
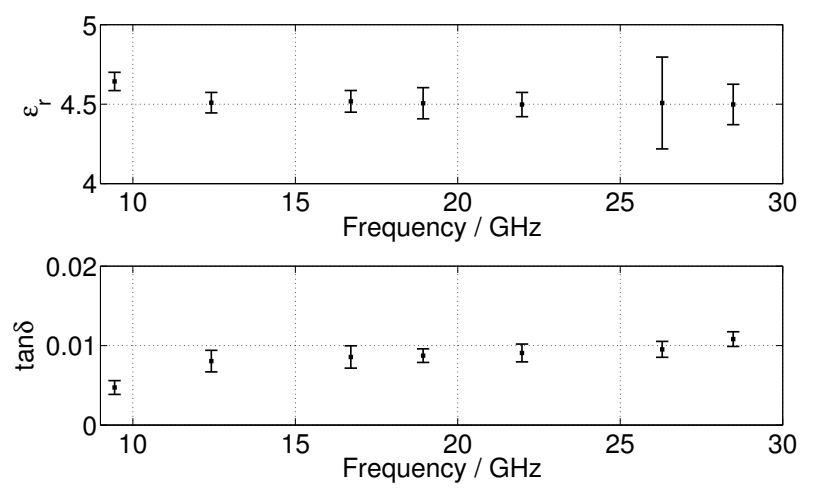

Figure 4. Measured relative permittivity and loss factor for BF33, $\varepsilon_{r} \approx 4.5, d=0.704 \mathrm{~mm}$.

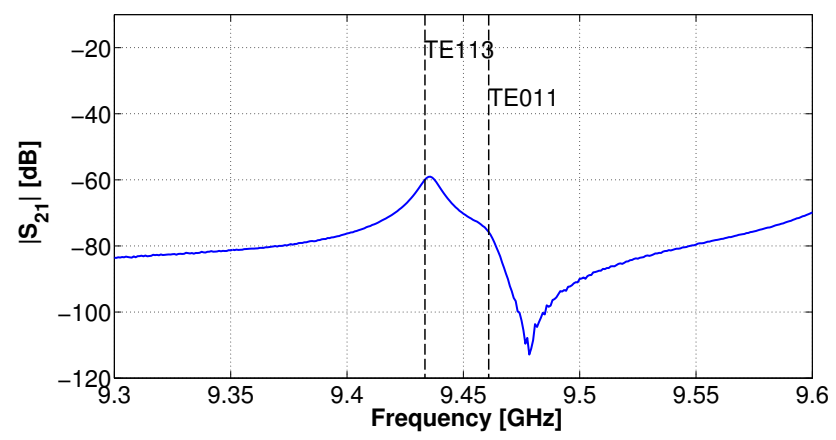

Figure 5. Measured spectrum with predicted $T E_{113}$ and $T E_{011}$ mode for BF33, $\varepsilon_{r} \approx 4.5, d=0.704 \mathrm{~mm}$.

\subsection{Sensitivity of modes and relative permittivity esti- mation}

To answer the question whether the relative permittivity of a substrate material can be estimated given a measured spectrum, a sensitivity analysis has been carried out for different modes by determining the change of the estimated relative permittivity as a function of the frequency variation around a certain frequency.

It is obvious from the results in Table 1 that odd $T E$ modes $(p=1,3, \ldots)$ are less sensitive to errors in the measured frequency $(\sim 0.01$ per $1 \mathrm{MHz})$ and thus more appropriate for relative permittivity estimation. On the other hand even $T E$ modes $(p=2,4, \ldots)$ show a huge sensitivity to frequency displacement $(\sim 10$ per $1 \mathrm{MHz})$ which makes them unapplicable for our purpose. The $T M$ modes lie in the middle range between odd and even $T E$ modes ( $\sim 0.1$ per $1 \mathrm{MHz}$ ), but due to their rare appearance they can in general be neglected.

Due to the finite range of relative permittivity values within which the estimation procedure is carried out, evaluating nearby resonance peaks in the measured spectrum can lead to estimation ambiguities in the pre-defined range. The results shown in Fig. 8 demonstrate three cases of estimation ambiguity concerning nearby resonances which could be 


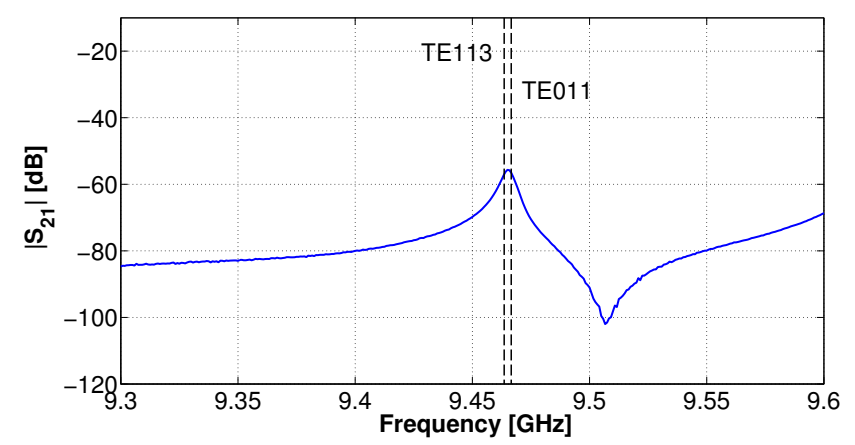

Figure 6. Measured spectrum with predicted $T E_{113}$ and $T E_{011}$ mode for $\mathrm{AF} 45, \varepsilon_{r} \approx 5.88, d=0.5 \mathrm{~mm}$.
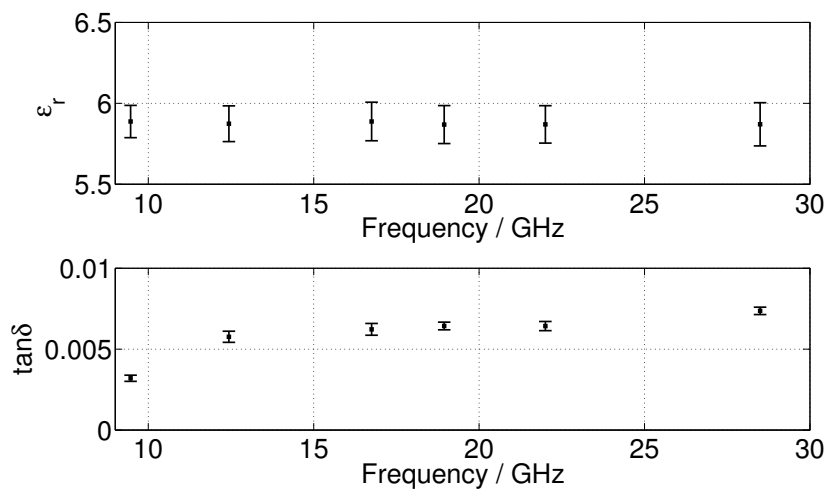

Figure 7. Measured relative permittivity and loss factor for AF45, $\varepsilon_{r} \approx 5.88, d=0.5 \mathrm{~mm}$.

encountered in practical measurements and therefore should be attended to.

In the first and most general case $(12.7150 \mathrm{GHz})$ each of the two applicable modes ( $T E_{411}$ and $T E_{121}$ ) leads to different estimates within the defined range at the two nearby resonance peaks. Here the two estimated values nearer to each other at the two resonance peaks can be chosen as the more likely estimates. In the second case $(9.4358 \mathrm{GHz})$ the absent peak of the $T E_{011}$ resonance in the measured spectrum (see Fig. 5) leads to two estimated values at the nearby $T E_{113}$ resonance peak, one for each of the predicted modes. In the third case $(14.5591 \mathrm{GHz})$ a single applicable mode $\left(T E_{115}\right)$ causes two different estimation values at two adjacent resonance peaks, one of which is related to the unapplicable (because of Table 1) even $T E_{122}$ mode. The estimation ambiguities in the last two cases can be solved by choosing the values nearer to a mean value determined by other modes as the more likely estimates and successively narrowing the estimation range. Figures 9 and 10 show estimation results for AF45 and alumina substrates, respectively.

In Table 2 estimation results of the extended model for odd $T E_{0 \text { np }}$ modes are compared to those estimated by the model presented in Janezic et al. (2004) for three substrate materials. The good agreement in the estimation results of
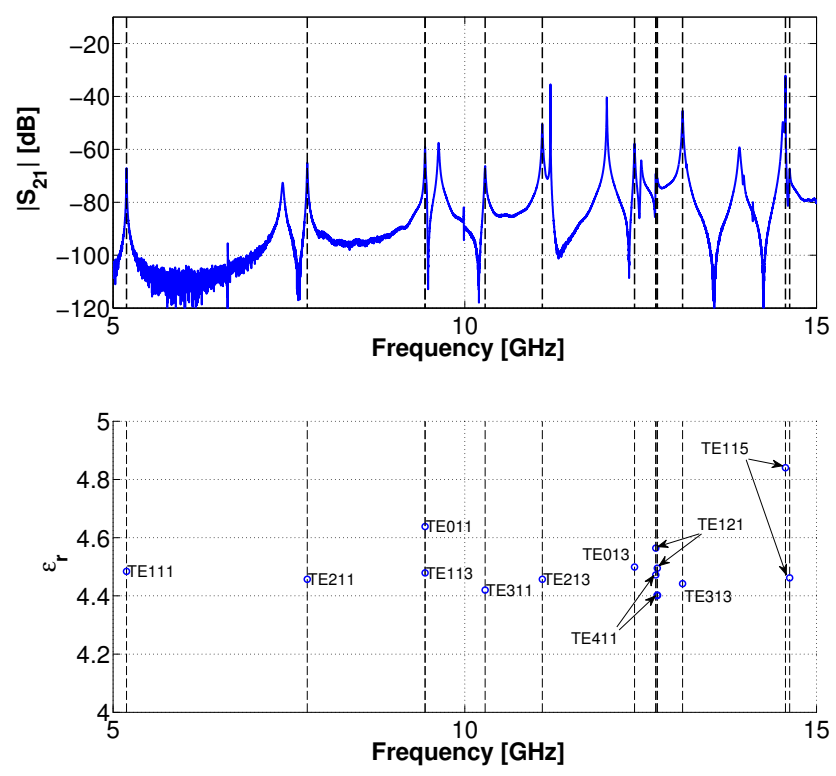

Figure 8. Measured spectrum and estimated relative permittivity in the frequency range $5-15 \mathrm{GHz}$ for BF33, $\varepsilon_{r} \approx 4.5, d=0.704 \mathrm{~mm}$.

Table 1. Sensitivity of estimated relative permittivity to resonance frequency variation $\left(\mathrm{BF} 33, \varepsilon_{r} \approx 4.5, d=0.704 \mathrm{~mm}\right)$.

\begin{tabular}{ccc}
\hline Mode & calc. freq. [GHz] & $\Delta \varepsilon_{r_{s}} / \Delta f_{\mathrm{MHz}}$ \\
\hline TE011 & 9.4608 & $9.0090 \mathrm{e}-003$ \\
TE012 & 11.2196 & $1.0180 \mathrm{e}+001$ \\
TE013 & 12.4131 & $7.2072 \mathrm{e}-003$ \\
TE111 & 5.1904 & $1.3514 \mathrm{e}-002$ \\
TE112 & 7.4233 & $1.5901 \mathrm{e}+001$ \\
TE113 & 9.4334 & $9.0090 \mathrm{e}-003$ \\
TM111 & 9.9865 & $9.8649 \mathrm{e}-002$ \\
TE114 & 12.5164 & $2.3423 \mathrm{e}+000$ \\
TE115 & 14.6122 & $6.3063 \mathrm{e}-003$ \\
TE121 & 12.7358 & $3.0503 \mathrm{e}-003$ \\
TE122 & 14.5605 & $7.9504 \mathrm{e}+000$ \\
TE211 & 7.7552 & $9.0090 \mathrm{e}-003$ \\
TE212 & 9.6085 & $1.2072 \mathrm{e}+001$ \\
TE213 & 11.0970 & $9.0090 \mathrm{e}-003$ \\
TE214 & 13.9238 & $2.0721 \mathrm{e}+001$ \\
TM212 & 13.9845 & $1.1532 \mathrm{e}-001$ \\
TE311 & 10.2733 & $4.5045 \mathrm{e}-003$ \\
TE312 & 12.0200 & $9.7072 \mathrm{e}+000$ \\
TE313 & 13.0888 & $7.2072 \mathrm{e}-003$ \\
TE411 & 12.7058 & $3.0603 \mathrm{e}-003$ \\
TE412 & 14.5286 & $7.9504 \mathrm{e}+000$ \\
\hline
\end{tabular}

both models confirms that the fringing fields in the gap region which are considered by the $T E_{0 \text { np }}$ model but neglected by our extended model do not affect the estimation for these modes considerably. However, loss tangent sensitivity to the fringing fields restricts our approach to relative permittivity extraction. 


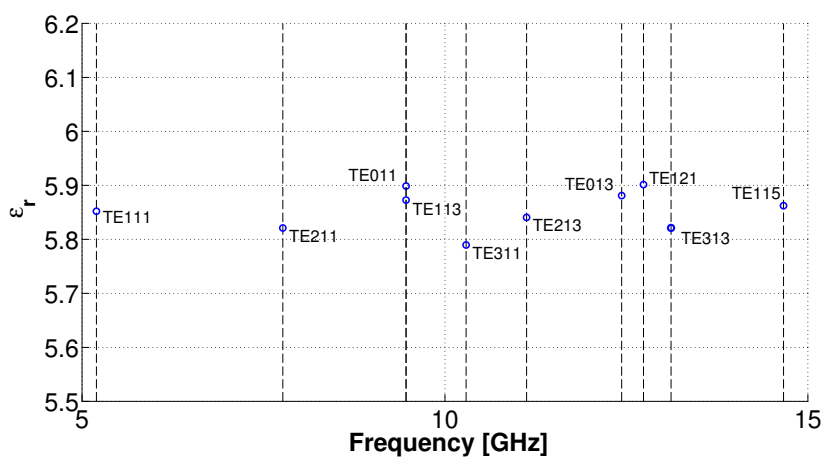

Figure 9. Estimated relative permittivity in the frequency range 5$15 \mathrm{GHz}$ for AF45, $\varepsilon_{r} \approx 5.88, d=0.5 \mathrm{~mm}$.

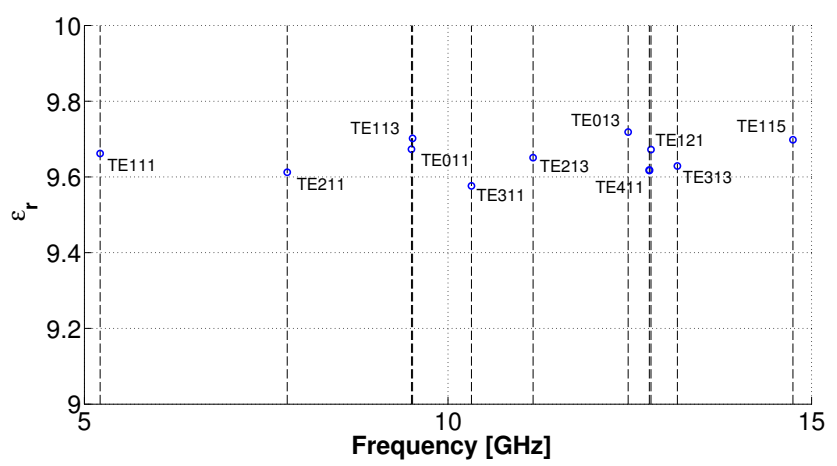

Figure 10. Estimated relative permittivity in the frequency range 5-15 GHz for alumina, $\varepsilon_{r} \approx 9.66, d=0.267 \mathrm{~mm}$.

\section{Conclusions}

We presented an extended mode-matching model enhancing the $T E_{0 \text { np }}$ mode-matching model presented in Janezic et al. (2004) by improving mode identification and enabling distortion analysis of resonant modes due to coupling and overlapping. Additionally a better frequency range coverage for relative permittivity estimation has been reached. The new model was verified by analyzing measured spectra of several substrate materials. Although our model does not consider the fringing fields in the gap region a comparison of relative permittivity estimation results for the $T E_{0 \text { np }}$ modes showed good agreement with the model of Janezic et al. (2004).

Acknowledgements. This work was funded by the European Metrology Research Programme (EMRP) under Grant Agreement No. 217257. The EMRP is jointly funded by the EMRP participating countries within EURAMET and the European Union.

Edited by: W. Mathis

Reviewed by: three anonymous referees
Table 2. Comparison between extended model and pure odd $T E_{0 \mathrm{np}}$ mode model estimation results for BF33 $(d=0.704 \mathrm{~mm}), \mathrm{AF} 45$ $(d=0.5 \mathrm{~mm})$ and Alumina $(d=0.267 \mathrm{~mm})$.

\begin{tabular}{|c|c|c|c|c|}
\hline & Mode & $\begin{array}{c}\text { meas. freq. } \\
{[\mathrm{GHz}]}\end{array}$ & $\begin{array}{c}\varepsilon_{r_{s}}(\text { extended } \\
\text { model })\end{array}$ & $\begin{array}{c}\varepsilon_{r_{s}}\left(T E_{0 \mathrm{np}^{-}}\right. \\
\text {model })\end{array}$ \\
\hline \multirow{6}{*}{$\stackrel{m}{m}$} & TE013 & 12.4133 & 4.499 & 4.509 \\
\hline & TE015 & 16.7075 & 4.498 & 4.517 \\
\hline & TE023 & 18.9325 & 4.504 & 4.505 \\
\hline & TE025 & 21.9758 & 4.486 & 4.497 \\
\hline & TE033 & 26.2908 & 4.509 & 4.507 \\
\hline & TE035 & 28.4766 & 4.496 & 4.498 \\
\hline \multirow{6}{*}{ 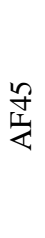 } & TE011 & 9.4641 & 5.899 & 5.887 \\
\hline & TE013 & 12.4350 & 5.881 & 5.874 \\
\hline & TE015 & 16.7483 & 5.892 & 5.887 \\
\hline & TE023 & 18.9433 & 5.877 & 5.868 \\
\hline & TE025 & 22.0008 & 5.880 & 5.869 \\
\hline & TE035 & 28.4932 & 5.865 & 5.870 \\
\hline \multirow{9}{*}{$\frac{\overparen{\Xi}}{\stackrel{\Xi}{\Xi}}$} & TE011 & 9.4983 & 9.673 & 9.634 \\
\hline & TE013 & 12.4775 & 9.718 & 9.689 \\
\hline & TE021 & 16.3566 & 9.673 & 9.636 \\
\hline & TE015 & 16.8200 & 9.729 & 9.724 \\
\hline & TE023 & 18.9699 & 9.683 & 9.679 \\
\hline & TE017 & 21.7846 & 9.747 & 9.748 \\
\hline & TE025 & 22.0516 & 9.733 & 9.713 \\
\hline & TE033 & 26.3066 & 9.737 & 9.692 \\
\hline & TE035 & 28.5233 & 9.746 & 9.719 \\
\hline
\end{tabular}

\section{References}

Ali, M. T., Salleh, M. K. M., and Zan, M. M. M.: Air-Filled Circular Cross Sectional Cavity for Microwave Non-Destructive Testing, in: Proceedings of World Academy of Science, Engineering and Technology, 18, 107-112, 2006.

Fang, X., Linton, D., Walker, C., and Collins, B.: A Tunable Split Resonator Method for Nondestructive Permittivity Characterization, IEEE T. Instrum. Meas., 53, 1473-1478, 2004.

Janezic, M.: Nondestructive Relative Permittivity and Loss Tangent Measurements Using a Split-Cylinder Resonator, PhD. thesis, University of Colorado at Boulder, 2003.

Janezic, M. D. and Baker-Jarvis, J.: Full-Wave Analysis of a SplitCylinder Resonator for Nondestructive Permittivity Measurements, IEEE T. Microw. Theory, 47, 2014-2020, 1999.

Janezic, M. D., Kuester, E. F., and Baker-Jarvis, J.: Broadband Complex Permittivity Measurements of Dielectric Substrates using a Split-Cylinder Resonator, IEEE MTT-S International Symposium Digest, 3, 1817-1820, 2004.

Janezic, M. D., Arz, U., Begley, S., and Bartley, P.: Improved Permittivity Measurement of Dielectric Substrates by use of the TE111 Mode of a Split-Cylinder Cavity, in: Proc. 73rd ARFTG IEEE Microwave Measurement Conference, 1-3, 2009.

Kent, G.: An evanescent-mode tester for ceramic dielectric substrates, IEEE T. Microw. Theory, 36, 1451-1454, 1988. 